\title{
Turbulent Vortex Ring/Free Surface Interaction
}

A. Weigand

M. Gharib

Graduate Aeronautical Laboratories, California Institute of Technology, Pasadena, CA 91125
The interaction of turbulent vortex rings that approach a clean water surface under various angles is experimentally investigated. The temporal evolution of the vortex rings with an initial Reynolds number of $R_{0}=7500$ is characterized by the laminar/ turbulent transition and asymptotic relaminarization of the flow. Using the shadowgraph technique, two major flow cases were identified as a result of the vortex-ring/ free-surface interaction: a trifurcation case that results from the interaction during the transition stage, and a bifurcation case that evolves during the fully-developed turbulent stage. In contrast to the laminar interaction, the turbulent bifurcation pattern is characterized by the reconnection and mutual interaction of many small-scale structures. Simultaneous digital particle image velocimetry (DPIV) and shadowgraph measurements reveal that the evolution of the small-scale structures at the free surface is strongly dominated by the bifurcation pattern, which in turn is a consequence of the persisting laminar sublayer in the core regions of the reconnected turbulent vortex loops.

\section{Introduction}

The interaction of complex shear flows (e.g., ship wakes) with a free surface is a very intriguing problem in fluid dynamics. Since full simulations of such complex flows are difficult to perform in the laboratory, free-surface interactions of elementary shear flows such as vortex rings and vortex couples have received much attention in the past. In this regard, the freesurface interaction of vortex rings and vortex couples was investigated mainly in the laminar flow regime. Bernal and Kwon (1989), Bernal et al. (1989), and Gharib et al. (1992) experimentally investigated the laminar vortex-ring/free-surface interaction. Bernal and Kwon showed that as a vortex ring approaches the free surface at an oblique angle, the upper part of the vortex ring deforms and opens its ends to reconnect to the surface. Additionally, Kwon (1989) observed that the lower part of the vortex ring can also interact with the free surface which results in the bifurcation of the vortex ring. Gharib et al. developed a physical flow model of the laminar vortex-ring reconnection including the effect of secondary vorticity that results from the local surface deformation and surface-tension differences.

In this paper, we mainly focus on the oblique interaction of turbulent vortex-rings with a free surface. Similar to experimental investigations of turbulent vortex/free-surface interactions (e.g., Sarpkaya et al., 1994), the major motivation for this study was to investigate the reconnection behavior of coexisting small- and large-scale structures in the presence of a highly three-dimensional flow field in the bulk. Additionally, in terms of the reconnection of closed vortex loops, turbulent vortexrings can be regarded as elemental flow structures that are part of more complex free-surface flows such as high Reynolds and Froude number ship wakes.

There is relatively little knowledge about the physical properties of turbulent vortex rings (see, e.g., Maxworthy, 1974 and Glezer and Coles, 1990). Therefore, we extensively utilized flow visualization and digital particle image velocimetry (DPIV) to map the initial conditions and the flow field of the vortex rings in the fully-submerged case (Weigand and Gharib, 1994). The interaction of the turbulent vortex rings with the

Contributed by the Fluids Engineering Division for publication in the JoURNAL OF Fluids ENGINEERING. Manuscript received by the Fluids Engineering Division October 17, 1994; revised manuscript received December 19, 1994. Associate Technical Editor: D. P. Telionis. free surface was visualized with the shadowgraph technique. Using combined shadowgraph and DPIV, simultaneous measurements of the deformation field and velocity-vector field at the water surface were performed.

\section{Experimental Setup and Procedures}

Experiments were conducted in a water tank filled with deionized water (D.I. water). Figure 1 shows a general schematic of the experimental setup. Vortex rings of diameter $D$, circulation $\Gamma$, and propagation velocity $U_{V}$ were generated by a piston that pushes fluid out of a sharp-edged cylindrical nozzle with an inner orifice diameter of $D_{0}=2.0 \mathrm{~cm}$. The nondeformed water surface coincides with the $x, z$-plane, where the positive $z$-axis points toward the reader. The centerline of the vortexring generator (i.e., the $x^{\prime}$-axis) is inclined with respect to the water surface at an angle $\alpha$, and the origin $x^{\prime}=0$ is located at $x=0$ and $y=-h$, where $h$ designates the submergence depth.

Several different procedures were applied to keep the water and its surface clean, and to minimize surface contamination and surface-tension differences between the water in the bulk and at the surface. Besides working in a clean environment (i.e., wearing non-contaminating gloves, cleaning all parts in contact with water using ethyl alcohol, operating a UV filter to prevent bacterial growth in the water, and exchanging the D.I. water every second or third day), a constantly operating skimmer and a vacuum operated suction device were used to remove the aging water surface. Surface-tension measurements were performed before and after each experimental run using a ring tensiometer. An IBM PC provides precise timing and synchronization of different events in the experiment (time resolution better than $10^{-3} \mathrm{~s}$ ). These events include vortex-ring generation, dye injection for flow visualization, and initialization of measurement processes.

Regarding DPIV, only those aspects of the measurement technique that are relevant to the present work are described in the following. For more details, the reader is referred to Willert and Gharib (1991). DPIV measures the two-dimensional displacement-vector field of particles that are suspended in the flow. As the schematic in Fig. 2 shows, the measurement plane is illuminated by a thin, pulsed sheet of laser light. In contrast to conventional particle image velocimetry, DPIV employs a video camera (RS 170 video standard) that records a sequence of images of the particle field. The camera is positioned normal to the illuminated measurement plane, while the exposure times 


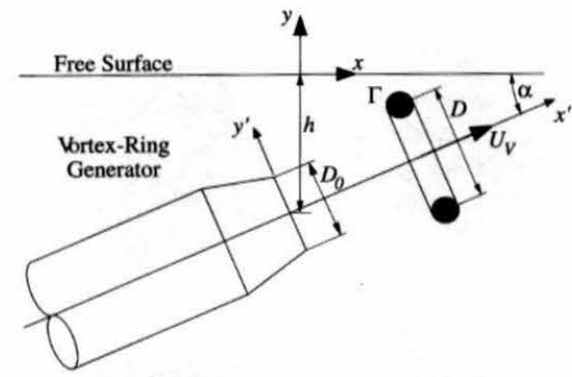

Fig. 1 General schematic of the experimental setup

and the time difference between two successive image exposures are synchronized and controlled by a camera controller and a shutter. The latter prevents streaking particle images and limits the maximum displacement of particles in the imaging plane. The recorded image sequence is stored on an analog video disk and subsequently digitized by a frame grabber. By cross-correlating spatial sub-samples (windows) of two successive video images, the average local displacement vector of the particles contained in the correlation window is estimated. Moving the correlation window over the entire image (moving average), the displacement-vector field is obtained. The latter is divided by the time difference of two successive image exposures yielding the velocity-vector field.

In Fig. 3, the experimental setup of the simultaneous DPIV and shadowgraph measurement technique is shown. Regarding the shadowgraph setup, a high power light source (Mercury arc-lamp, $1000 \mathrm{~W}$ ) is used in combination with a $20 \mathrm{~cm}$ ( 8 in) diameter spherical mirror $(\mathrm{F \#}=3)$ to generate a parallel beam of white light. The beam is reflected through the water surface by a front surface mirror (M2). A second front surface mirror (M3) projects the refracted light beam on a diffusive screen, where the shadowgraph images are recorded by a shuttered video camera. With respect to the velocity-field measurements, the DPIV camera focuses via mirror M2 through the bottom of the water tank onto the measurement plane. Therefore, refractive distortions of the particle-scatter light caused by the deformation of the water surface are eliminated. The measurement plane is illuminated by a $0.1 \mathrm{~cm}$ thick sheet of laser light that is positioned parallel and $0.1 \mathrm{~cm}$ below the water surface. A narrow band-pass filter (bandwidth: $\pm 5 \mathrm{~nm}$ ) centered on the green Argon-Ion laser line $(514.5 \mathrm{~nm})$ prevents the DPIV camera from recording scatter light of the shadowgraph illumination. To obtain a large overlapping field of view of both cameras, the parallax angle of $\Theta \approx 3.5^{\circ}$ was kept small by positioning the spherical mirror M1 and the DPIV camera close to each other and at a relatively large distance of $2.5 \mathrm{~m}(8 \mathrm{ft})$ from

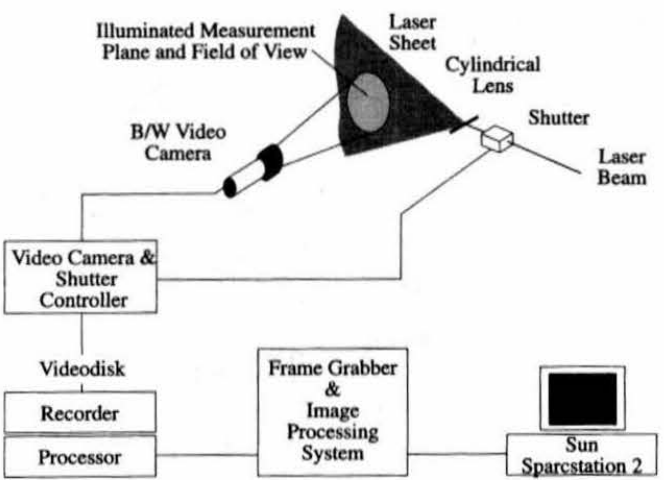

Fig. 2 Digital particle image velocimetry (DPIV) system

mirror M2. As Fig. 4 shows, the exposure of the shuttered shadowgraph camera is centered between the two successive illumination peaks of the DPIV measurement. Therefore, the shadowgraph and DPIV measurements are truly simultaneous.

In the present experiments, neutrally buoyant, silver-coated glass spheres with an average diameter of $14 \pm 5 \mu \mathrm{m}$ were used as seeding particles. The DPIV images were digitized with a resolution of $768 \times 480$ pixels, and processed with a window size of $32 \times 32$ pixels and a step size for the moving average of $8 \times 8$ pixels ( 75 percent window overlap). The data processing results in a field measurement of $96 \times 60$ velocity vectors and, for a typical field of view of $11 \times 8 \mathrm{~cm}$, in a spatial wavelength resolution of $0.46 \times 0.53 \mathrm{~cm}$. In the fully-submerged flow case, the exposure times and time difference between exposures were chosen to be $t_{e 1}=t_{e 2}=2 \cdot 10^{-3} \mathrm{~s}$ and $\Delta t=6 \cdot 10^{-3} \mathrm{~s}$. The simultaneous DPIV and shadowgraph measurements required a different illumination setting with $t_{e 1}=t_{e 2}=11 \cdot 10^{-3} \mathrm{~s}, \Delta t=$ $17 \cdot 10^{-3} \mathrm{~s}$, and $t_{e s}=4 \cdot 10^{-3} \mathrm{~s}$. The resulting displacementvector fields were geometrically corrected for the parallax error, while the relatively large exposure times of the DPIV camera were chosen in order to compensate for the loss of particlescatter light in the bandpass filter. In the present results, the maximum surface velocities are smaller than $5 \mathrm{~cm} / \mathrm{s}$. Therefore, the maximum particle displacement was approximately 6 to 8 pixels, and the size of streaking particle images was limited to less than 4 pixels. Since the location of the cross-correlation peak can be resolved with a sub-pixel accuracy of better than 0.01 pixel (Willert and Gharib, 1991), the maximum uncertainty based on the local velocity and vorticity magnitude is \pm 1 and \pm 3 percent, respectively.

\section{Properties of Turbulent Vortex Rings}

Prior to the investigation of the vortex-ring/free-surface interaction, the evolution of the vortex rings was investigated in

\section{Nomenclature}

$D=$ diameter of vortex-ring

$D_{0}=$ inner diameter of cylindrical nozzle

$\mathrm{Fr}=$ Froude number $; \mathrm{Fr}=\Gamma /\left(g D^{3}\right)^{1 / 2}$

$g=$ gravitational acceleration; $g=$ $9.81 \mathrm{~m} / \mathrm{s}^{2}$

$h=$ submergence depth of vortex-ring generator

$n=$ wavenumber of bending instability

$\mathrm{Re}=$ Reynolds number of vortex ring; $\operatorname{Re}=\Gamma / \nu$

$\mathrm{Re}_{0}=$ initial Reynolds number of vortex ring

$t=$ time; $t=0$ : initiation of piston motion $t_{e}=$ frame-exposure time

$\Delta t=$ time difference between exposures of DPIV measurements

$U_{V}=$ propagation velocity of vortex ring

$\mathrm{We}=$ Weber number; We $=\rho \Gamma^{2} /$ $(\sigma D)$

$x, y, z=$ Cartesian coordinate system; $y=0$ : location of non-deformed water surface

$x^{\prime}, y^{\prime}, z^{\prime}=$ Cartesian coordinate system fixed to vortex-ring generator ; $x^{\prime}=0$ : location of nozzle center $\alpha=$ angle between centerline of vortexring generator and free surface

$\Gamma=$ circulation of vortex ring

$\Gamma_{0}=$ initial circulation of vortex ring

$\nu=$ kinematic viscosity of water $; \nu=$ $10^{-2} \mathrm{~cm}^{2} / \mathrm{s}$

$\rho=$ density of water; $\rho=1000 \mathrm{~kg} / \mathrm{m}^{3}$

$\sigma=$ surface tension of water; $\sigma=72.1$ $\pm 0.5 \mathrm{dyn} / \mathrm{cm}\left(1 \mathrm{dyn}=10^{-5} \mathrm{~N}\right)$

$\omega=$ vorticity component (indices $x, y, z$ indicate direction)

$\Theta=$ parallax angle between DPIV and shadowgraph camera 


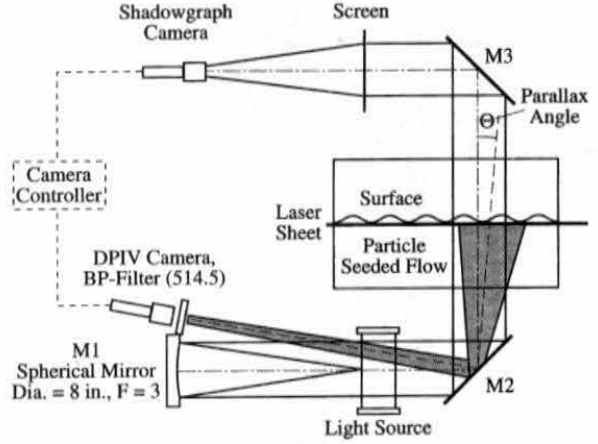

Fig. 3 Experimental setup of the simultaneous DPIV and shadowgraph technique

the fully-submerged case by means of flow-visualization and digital particle image velocimetry (DPIV). In the following, some major results of this study are reported for a flow case with initial Reynolds number of $\mathrm{Re}_{0}=7500$ (for a more detailed discussion, see Weigand and Gharib, 1994). The Reynolds number is defined by the ratio of the circulation $\Gamma$ to the kinematic viscosity $\nu$, i.e., $\operatorname{Re}=\Gamma / \nu$.

The image sequence shown in Fig. 5 indicates the important stages of the temporal evolution of the vortex ring. The images are front views (along the negative $x^{\prime}$-axis) and were realized by using dye-flow visualization. Time $t=0$ corresponds to the initiation of the piston motion. As Fig. 5-I shows, during the generation and for an initial period of $t \approx 1.3 \mathrm{~s}$, the vortex ring is characterized by a laminar flow behavior. After $t \approx 1.3 \mathrm{~s}$, azimuthal bending instabilities similar to those observed by Krutzsch (1936, 1939), Widnall and Sullivan (1973), and Widnall et al. (1974) start to develop which marks the beginning of the transition stage. In Fig. 5-II and III, the azimuthal bending instability with a wave number of $n=8$ leads to a strong and symmetric growth of the ring deformation within a short period of approximately $0.3 \mathrm{~s}$. As Fig. 5-IV shows, the latter causes a sudden break up of the vortex ring at $t \approx 2.5 \mathrm{~s}$ which marks the beginning of the turbulent stage. As the side-view (along the negative $z$-axis) in Fig. 6 indicates, the turbulent stage is characterized by a strong dye-shedding process into the wake of the vortex ring.

In Fig. 7, DPIV measurements of the temporal evolution of the vortex ring are presented. The measurement plane coincides with the plane $z=0$. Figure $7(a)$ shows the evolution of the magnitude of the circulation that was computed from the line integral of the velocity data along the vorticity contour $\omega_{z}=$ $\pm 2.5 \mathrm{~s}^{-1}$. As the experimental investigations of Willert and Gharib (1991) show, the uncertainty in the circulation measure-

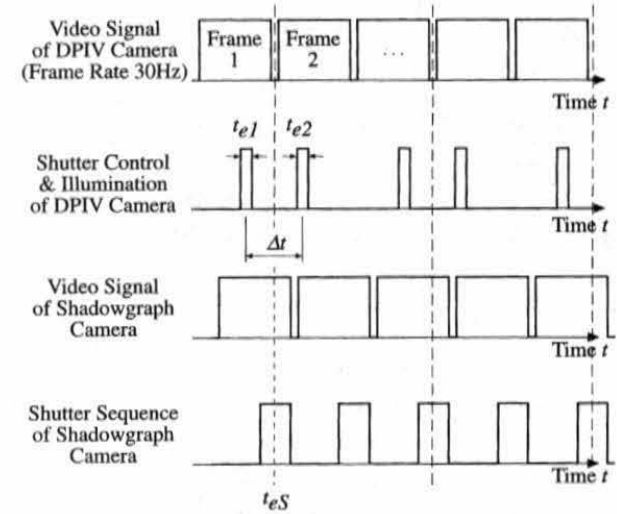

Fig. 4 Illumination and exposure sequence of the synchronized DPIV and shadowgraph cameras

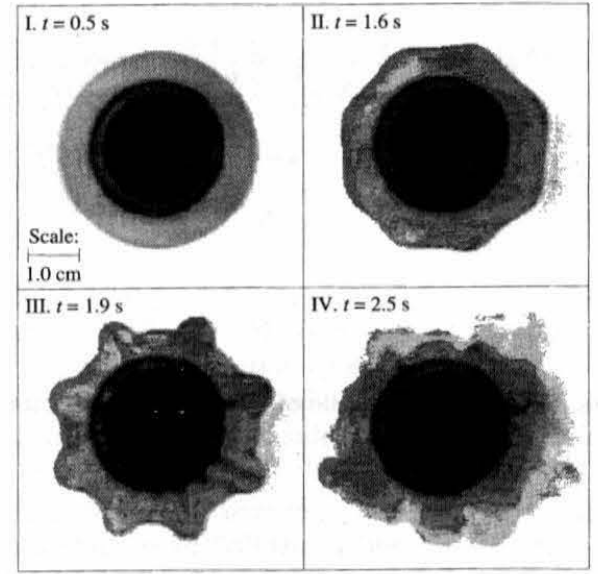

Fig. 5 Sequence of front views (along the negative $x^{\prime}$-axis) of the evolution of a turbulent vortex ring with $\mathrm{Re}_{0}=\mathbf{7 5 0 0}$

ment is better than \pm 1 percent of the local circulation value. The vorticity contour $\omega_{z}= \pm 2.5 \mathrm{~s}^{-1}$ separates the upper- and lower-core regions from the ambient flow field and the wake region. In order to cover the spatial extension of the flow, the evolution of the flow field was measured in seven consecutive and partially overlapping downstream regions. The circulation data is averaged over four independent realizations and was found to be repeatable within \pm 2 percent of the presented average values. The solid line represents the mean value of the magnitude of the upper and lower core circulation. In Fig. 7(b)I to IV, equi-vorticity contours of instantaneous flow fields that correspond to the major stages of the evolution of the vortex ring are shown. The vorticity field with $\omega_{z}=\partial v / \partial x-\partial u / \partial y$ was computed by using a second order finite differencing scheme and smoothed with a $3 \times 3$ radially weighting smoothing kernel.

During the initial stage, the nearly circular shape and dense distribution of vorticity contours at $t=0.5 \mathrm{~s}$ indicate the laminar and highly concentrated core structure of the vortex ring with peak vorticities of $\omega_{z} \approx \pm 80 \mathrm{~s}^{-1}$. Shortly after the transition stage, the core structure in Fig. 7(b)-II shows first signs of the turbulent break up leading to a relatively mild reduction in the circulation of approximately 8 percent between $1.6 \mathrm{~s}<t<$ $2.5 \mathrm{~s}$. Additionally, the shape of the upper and lower core is symmetrically deformed, and its size has increased which is indicated by a 25 percent reduction of the peak vorticities. At $t \approx 3 \mathrm{~s}$, the on-going break up leads to a further reduction of the circulation due to the shedding of vorticity into the wake of the vortex ring.

Figure $7(a)$ shows that the circulation does not decrease monotonically, but in periodic stages with intermittent and nearly constant levels of circulation. In combination with the vorticity-contours in Fig. $7(b)$-III, the latter is a remarkable indication of the periodic shedding process of small-scale struc-

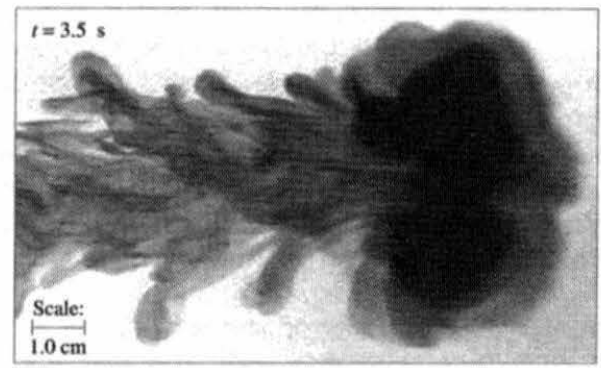

Fig. 6 Side view (along the negative $z^{\prime}$-axis) of a turbulent vortex ring with $\mathbf{R e}_{0}=\mathbf{7 5 0 0}$ after the transition stage 


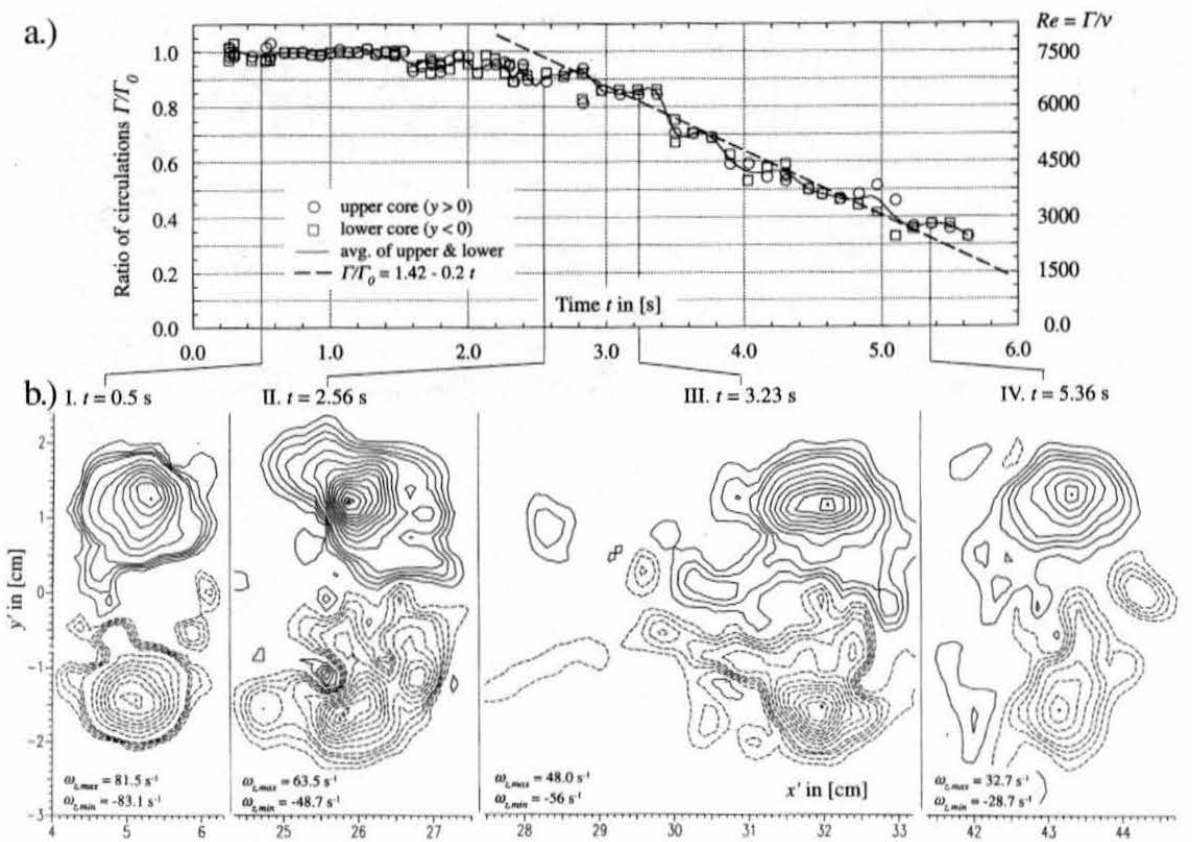

Fig. 7 Temporal evolution of a turbulent vortex ring with $R_{e_{0}}=7500$ : (a) circulation; (b) sequence of vorticity fields; frame I: $\Delta \omega_{z}= \pm 2.5 \mathrm{~s}^{-1}$ for $2.5 \mathrm{~s}^{-1} \leq\left|\omega_{z}\right| \leq 10 \mathrm{~s}^{-1}, \Delta \omega_{z}= \pm 10 \mathrm{~s}^{-1}$ for $\left|\omega_{z}\right|>10 \mathrm{~s}^{-1}$; frames II to IV: $\Delta \omega_{z}= \pm 2.5 \mathrm{~s}^{-1}$ for $2.5 \mathrm{~s}^{-1} \leq\left|\omega_{z}\right| \leq 10 \mathrm{~s}^{-1}, \Delta \omega_{z}= \pm 5 \mathrm{~s}^{-1}$ for $\left|\omega_{z}\right|>10 \mathrm{~s}^{-1}$

tures that contain a finite amount of circulation. Between $2.6 \mathrm{~s}$ $\leq t \leq 5.6 \mathrm{~s}$, the time averaged decrease of the vortex-ring circulation is best described by the linear function $\Gamma / \Gamma_{0}=1.42$ $-0.2 t$. Figure $7(b)$-III indicates that, even though the vortex ring is in the break-up stage, the vorticity distribution in the core regions remains relatively concentrated. After the vortex ring lost approximately 65 percent of its initial circulation to the wake, Fig. 7(b)-IV shows at $t=5.4 \mathrm{~s}$, that the core structures are still persistent and regularly shaped. This indicates a fourth and asymptotic stage of the temporal evolution of the vortex ring, namely the relaminarization of the flow. In general, the loss of circulation is equivalent to a reduction of the Reynolds number of the vortex ring. In the case presented here, the Reynolds number decreases from the initial value of $\mathrm{Re}_{0}=$ 7500 to $\operatorname{Re} \approx 2300$ at $t=5.6 \mathrm{~s}$.

\section{Turbulent Vortex-Ring/Free-Surface Interaction}

Overview. As the results of Section 3 indicate, the vortex ring can start to interact with the water surface at different stages, namely during the laminar, transition or fully turbulent stage. By varying the angle $\alpha$ and the submergence depth $h$ of the vortex-ring generator in a range of $7 \leq \alpha \leq 20 \mathrm{deg}$ and $2 D_{0} \leq h \leq 3 D_{0}$, the three interaction cases were investigated using a vortex ring with an initial Reynolds number of $\mathrm{Re}_{0}$ $=7500$. The Froude and Weber number based on the initial conditions are $\mathrm{Fr}=\Gamma /\left(g D^{3}\right)^{1 / 2}=0.46$ and $\mathrm{We}=\rho \Gamma^{2} /(\sigma D)$ $=26$, with the initial diameter of the vortex ring $D=3.0 \mathrm{~cm}$, the measured surface tension of D.I.-water $\sigma=72.1 \pm 0.5 \mathrm{dyn} /$ $\mathrm{cm}$, the density of water $\rho=1000 \mathrm{~kg} / \mathrm{m}^{3}$, and the gravitational acceleration $g=9.81 \mathrm{~m} / \mathrm{s}^{2}$.'

${ }^{1}$ The surface tension measurements with a ring tensiometer (nominal accuracy of $\pm 0.1 \mathrm{dyn} / \mathrm{cm}$ and experimentally determined repeatability of $\pm 0.5 \mathrm{dyn} / \mathrm{cm}$ ) revealed that the efforts to keep the water surface clean practically prevented surface contamination. The latter is also indicated by the presented shadowgraph images, where the formation of Reynolds ridges due to surface-tension differences was not observed. Regarding DPIV measurements, the particle seeding with silvercoated glass spheres and a concentration in the order of $10^{3}$ to $10^{4} \mathrm{~cm}^{-3}$ had no measurable effect on the magnitude of the surface tension.
Interaction During the Transition Stage. The sequence of shadowgraph images in Fig. 8( $a$ ) shows the temporal evolution of the flow at the free surface for a vortex ring that starts to interact with the free surface during the transition stage. This case with $h=3.8 \mathrm{~cm}$ and $\alpha=7 \mathrm{deg}$ leads initially to the bifurcation of the vortex ring. The bifurcation pattern is very similar to the results reported by Kwon (1989) who investigated the free-surface interaction of laminar vortex rings with a Reynolds number of $\operatorname{Re}=5000$ and an incidence angle of $\alpha=20$ deg. The schematic in Fig. $9(a)$ shows the major stages of the vortex-ring bifurcation. Initially, the interaction with the free surface leads to the reconnection of the upper part of the vortex ring and the formation of a single, $\mathrm{U}$-shaped vortex loop. Due to the impulse of the ring and the oblique angle with the free surface, the lower part of the vortex loop moves upward and, in the case of a clean surface, reconnects too. The latter leads to the splitting of the lower part of the vortex ring and the symmetric formation of two separate, U-shaped vortex loops. As frame I in Fig. 8( $a$ ) shows, the bifurcation process evolves in a regular fashion without the superposed reconnection of small-scale structures. Additionally, downstream of the bifurcation, a very distinct and regular wave pattern becomes visible that is not a result of imperfections in the initial conditions (e.g., mechanical vibrations or wave reflections caused by the tank walls). This pattern is very similar to a wave-field that is generated by the superposition of two closely aligned point sources. Upstream of the bifurcation, the surface-deformation field is characterized by waves that are aligned parallel to the $x$-axis. The latter is also observed during the interaction of vortex pairs with a free surface (see, e.g., Sarpkaya, 1992). As the reconnection of small-scale structures in frame II at $t=3.2$ $\mathrm{s}$ indicates, the bifurcated and separated vortex loops break up simultaneously and leave behind a strong and elongated signature of their wake at the free surface. The latter is caused by longitudinal vortices that form during the initial stage of the vortex-ring break-up due to the strong growth of the bending deformations. The evolution of these longitudinal vortices in the wake of the bifurcated vortex loops leads to their simultaneous and symmetric reconnection at the free surface. Therefore, 

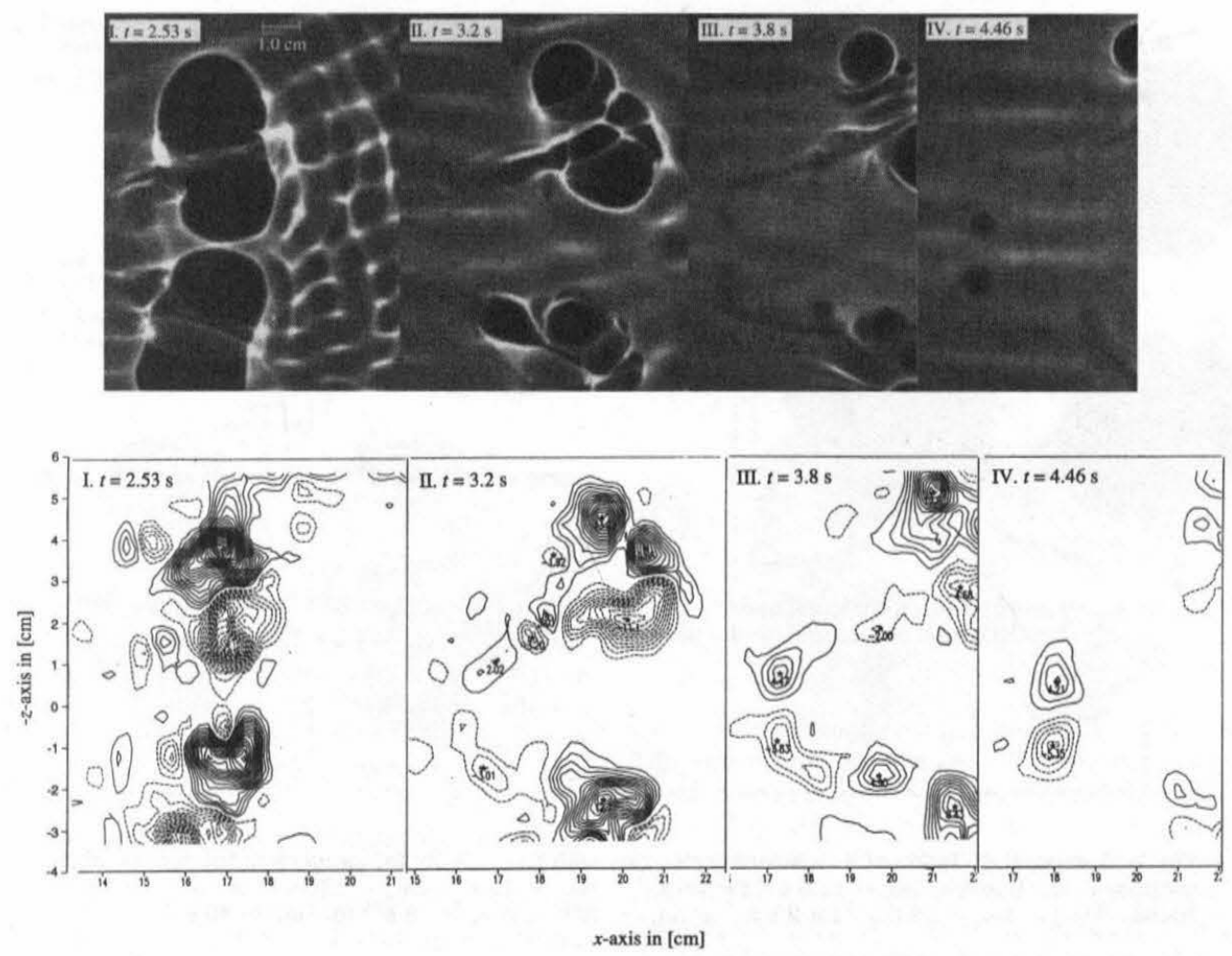

Fig. 8 Simultaneous DPIV and shadowgraph measurements of the reconnection of a vortex ring during the transition stage with $\mathrm{Re}_{0}=7500, h=3.8 \mathrm{~cm}$ and $\alpha=7^{\circ}:(a)$ sequence of shadowgraph images; (b) corresponding sequence of vorticity-fields; first vorticity contour $\omega_{y}= \pm 1 \mathrm{~s}^{-1}$, step size $\Delta \omega_{y}= \pm 1 \mathrm{~s}^{-1}$

as frames III and IV indicate, a third vortex pair appears upstream and centered between the bifurcated vortex loops which leads to a trifurcation pattern at the surface (see the schematic in Fig. $9(b))$. This process was observed to be very typical and also repeatable for the vortex-ring/free-surface interaction during the transition stage.

Figure $8(b)$ shows DPIV measurements of the flow field close to the free surface in the plane $y=-0.1 \mathrm{~cm}$ that are simultaneous with the shadowgraph images of Fig. 8( $a)$. Since the spatial scale of Figs. 8( $a)$ and $8(b)$ is the same, the vorticity ( $\omega_{y}$-component $)$ and deformation field can be easily compared by superposing both image sequences. As frame I in Fig. 8(b) shows, the initial bifurcation pattern is clearly marked by two concentrated and reconnected vortex pairs. The spatial location and the size of the vortex pairs agrees very well with the defor-

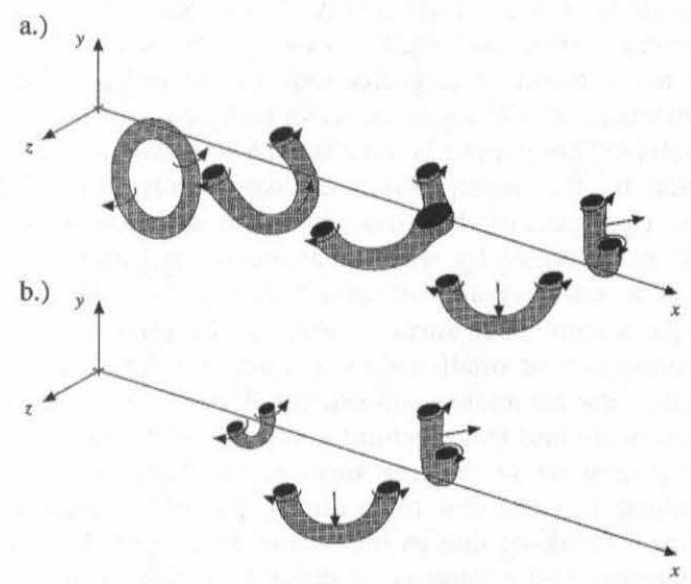

Fig. 9 Schematics of the major stages of $(a)$ vortex-ring bifurcation and (b) transitional vortex-ring trifurcation mation pattern shown in the corresponding shadowgraph image of Fig. 8( $a)$-I. In Fig. 8(b)-II, elongated vorticity regions upstream of the vortex pairs indicate the initial interaction of the longitudinal wake vortices with the free surface. Additionally, in partial agreement with the deformation field, the turbulent break up of the reconnected vortex loops is characterized by the formation and reconnection of small-scale vortices at the free surface. The reason for deviations between the deformation and the vorticity field in terms of the signature of small-scales at the free surface is twofold: first, the structures that are visible, for example, upstream of the bifurcated vortex pair in the lower part of the shadowgraph image of Fig. 8(a)-II are partially too small to be resolved by the DPIV measurements. Instead, the vorticity field in Fig. 8(b)-II indicates a comparatively large, negative vorticity region. Second, and more important in a physical sense, the deformation field is not only affected by the vortical regions, but also by the irrotational velocity field at the surface (see, e.g., Dommermuth, 1992). The latter is visible, for example, in frame I of Figs. $8(a)$ and $8(b)$, where the wave field upstream of the bifurcation pattern does not leave any signature in the vorticity field.

In frame III of Fig. 8(b), the vorticity field clearly shows the concentrated and symmetric reconnection of the wake vortices which leads to the formation of a third vortex pair and the eventual trifurcation pattern at the free surface. In contrast to the vorticity field, the shadowgraph image in Fig. 8(a)-III shows only a faint signature of the initial stage of the trifurcation pattern. The latter is marked by the curl-like shape of the surface deformation at the upstream end of the strongly elongated wake vortices. In good agreement with the deformation field, Fig. $8(b)$-IV shows the further separation and concentration of the third vortex pair upstream of the bifurcated vortex loops.

Interaction During the Fully-Developed Turbulent Stage. In Fig. 10, the sequence of shadowgraph images shows the typical evolution of a fully-developed, turbulent vortex-ring/ 


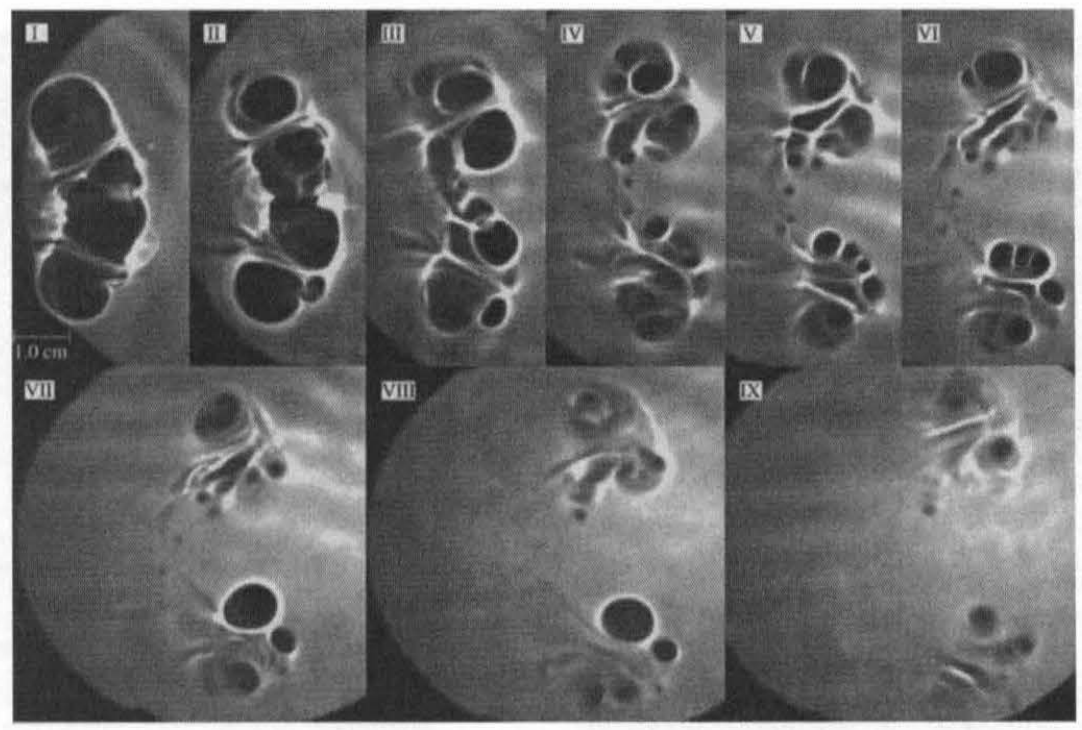

Fig. 10 Shadowgraph sequence of the reconnection of a fully-developed turbulent vortex ring with $\mathrm{Re}_{0}=7500, h=5.4 \mathrm{~cm}$ and $\alpha=7^{\circ}$; frame I: $t=3.3 \mathrm{~s}$, time difference between successive frames: $0.33 \mathrm{~s}$

free-surface interaction with $h=5.4 \mathrm{~cm}$ and $\alpha=7 \mathrm{deg}$. The single frames are separated by a time difference of $\Delta t=0.33$ $\mathrm{s}$. As Frame I at time $t=3.3 \mathrm{~s}$ shows, the initial stage of the interaction is marked by a strong surface deformation that is caused by the reconnection of the upper part and the simultaneous upward motion of the lower part of the vortex ring. Between frame II and IV, the reconnection of the lower part leads to the bifurcation of the vortex ring into two separate and perpendicularly reconnected vortex loops at the free surface. However, in contrast to the transition case, the shadowgraph images indicate the early and multiple reconnection of smaller-scale structures as a consequence of the shedding process of the fully-developed turbulent vortex ring. The dynamics of the small-scale structures at the free surface appears to be dominated by the bifurcation pattern. However, as many observable pairing processes show, the small-scale structures interact strongly with each other within that bifurcation pattern. For example, in the lower part of the shadowgraph images between frame III and VII, four distinct small-scale structures reconnect to the free surface, of which at least three are subject to a very rapid pairing process. The triple pairing evolves in less than $0.33 \mathrm{~s}$ between frame VI and VII and leads to a concentrated and relatively large-scale single vortex. The latter is indicated by the strong and circular surface deformation in the lower part of frames VII and VIII.

Figures 11 $(a)$ to $11(c)$ show a typical example of simultaneous DPIV and shadowgraph measurements of the vortex-ring/ free-surface interaction during the fully-developed turbulent stage. The figure shows the evolution of the turbulent bifurcation at the free surface in the half plane $z \leq 0$. The initial conditions are the same as in the flow case presented in Fig. 10. In order to compare the spatial correspondence, the resulting vorticity and deformation fields are superposed to each other (the gray scale of the shadowgraph images was slightly modified to enhance the visibility of the vorticity contours).

Figure 11 $(a)$ shows the initial and distinct deformation pattern at the surface consisting of the bifurcated vortex pair superposed by several small-scale structures. For example, in the center and the upper part of the vortex pair, the separated smallscale dark regions of the shadowgraph image are in excellent spatial agreement with small-scale vortical regions. In the lower part of Fig. 11 $(a)$ the large circular deformation corresponds to a relatively large and concentrated vortex. Additionally, in the wake of the vortex pair, the flow field at the surface consists of several small-scale vortical structures that are not visible as single structures in the deformation field. Following the temporal evolution of the vorticity and deformation field, Fig. 11(b) indicates that the small-scale structures spatially align according to the flow field that is induced by the bifurcated and relatively strong vortex pair. In comparison to Fig. 11 $(a)$, the vortex pair still leads to a visible but weaker surface deformation. The latter is also indicated in the vorticity field, where the scale of the major vortex pair increases and the magnitude of the peak vorticity decreases.

Figure 11 $(c)$ shows that the upper vortex re-concentrates its vorticity distribution which is indicated by the dense distribution of equi-vorticity contours, a relatively high peak vorticity, and a strong surface deformation. In contrast to that, the size of the lower vortex is increased and its peak vorticity reduced yielding a relatively weak surface deformation. In comparison to the earlier stages, Fig. 11 (c) shows that the number of reconnected small-scale structures has considerably reduced. The latter is a result of the combined action of different mechanisms, e.g., the loss of structures to the wake and the underlying threedimensional flow, and pairing processes at the free surface similar to those shown in Fig. 10.

In comparison to the development of the fully-submerged turbulent vortex ring, Fig. 12 shows the temporal evolution of the total positive and negative circulation $\Gamma_{+}$and $\Gamma_{-}$that reconnects to the free surface within the boundaries of the measurement region (i.e., in the half-plane $z \leq 0$ ). Within the regarded time interval, the flow behavior at the surface is symmetric with respect to the magnitudes of the total circulations, i.e., $\left|\Gamma_{+}\right|=\left|\Gamma_{-}\right|$. The latter indicates that all reconnection and disconnection events within the measurement region are accounted for. As Fig. 12 shows, the magnitude of the reconnected total circulation in the half plane $z \leq 0$ is approximately 70 percent of the vortex-ring circulation in the fullysubmerged case. As is evident from the schematics in Fig. 9( $a)$, in the case of a laminar vortex-ring bifurcation, the reconnected vortex pair can be expected to have the same amount of circulation as the fully-submerged vortex ring. In the case of a turbulent bifurcation, however, it is not clear at this point, why only 70 percent of the vortex-ring circulation are detected at the free surface. One can conjecture that the three dimensional flow field in the bulk prevents part of the small-scale structures from reconnecting to the surface. On the other hand, the underlaying flow field might cause multiple folding, bending, and reconnection processes of single vortex tubes leading to an increase 

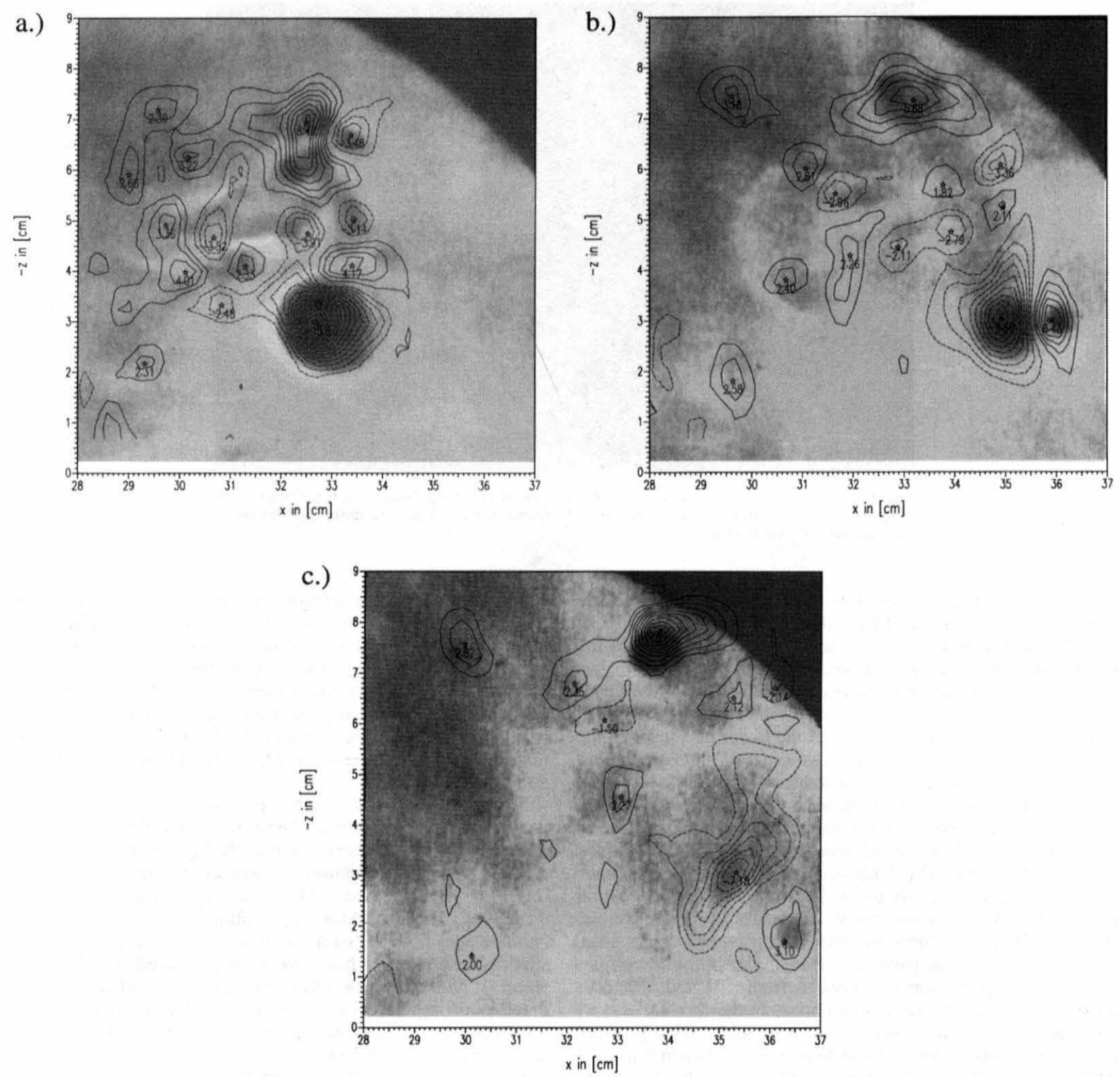

Fig. 11 Overlay sequence of simultaneous DPIV and shadowgraph measurements for the reconnection of a fully-developed turbulent vortex ring with $\mathrm{Re}_{0}=7500, h=5.4 \mathrm{~cm}$ and $\alpha=7^{\circ}$; first vorticity contour $\omega_{y}= \pm 1 \mathrm{~s}^{-1}$, step size $\Delta \omega_{y}= \pm 1 \mathrm{~s}^{-1} ;(a) t=5.2 \mathrm{~s},(b) t=6.2 \mathrm{~s}$, and $(c) t=6.9 \mathrm{~s}$

of the circulation at the surface. However, the limited spatial resolution of the measurement technique makes it experimentally difficult to detect and separate the effects of the bulk flow on the reconnection behavior of smaller-scale structures.

To gain more information about the mechanisms that are of importance for the flow behavior at the free surface, the measurement sequence in Fig. 11 was used to track the development of the small-scale structures and their associated circulations. In Fig. 13, splitting and pairing as well as reconnection and disconnection events are marked by different symbols. At time $t \approx 4.7 \mathrm{~s}$, the regarded flow field contains four separate structures, while at $t \approx 5.2 \mathrm{~s}$, three additional small-scale vortices reconnect to the surface. For example, at $t \approx 5.5 \mathrm{~s}$, the structure with a circulation of $\Gamma \approx 19 \mathrm{~cm}^{2} / \mathrm{s}$ was formed by a triple pairing event and separates into three new structures due to a splitting event. During the regarded time interval, a total of ten pairing and ten splitting events were observed, which also includes the pairing of opposite sign vorticity, i.e. the can- cellation of vorticity at the free surface. As Fig. 13 shows, pairing and splitting events can be observed on all measured scales. Between $5.5 \mathrm{~s} \leq t \leq 6.9 \mathrm{~s}$, the number of small-scale structures is reduced from seven to four, while the number of large-scale structures (major vortex pair) remains constant.

\section{Conclusions}

Regarding the vortex-ring/free-surface interaction, two distinct flow cases were observed. During the interaction in the transition stage, the vortex ring initially bifurcates into two separate vortex pairs at the free surface, while the longitudinal wake vortices of the bifurcated vortex loops simultaneously reconnect and form a third vortex pair at the free surface. During the evolution of this trifurcation case, the bifurcated and reconnected vortex loops break up at the free surface which leads to the subsequent reconnection of small-scale structures. Similar to the transition case, the interaction in the fully-developed 


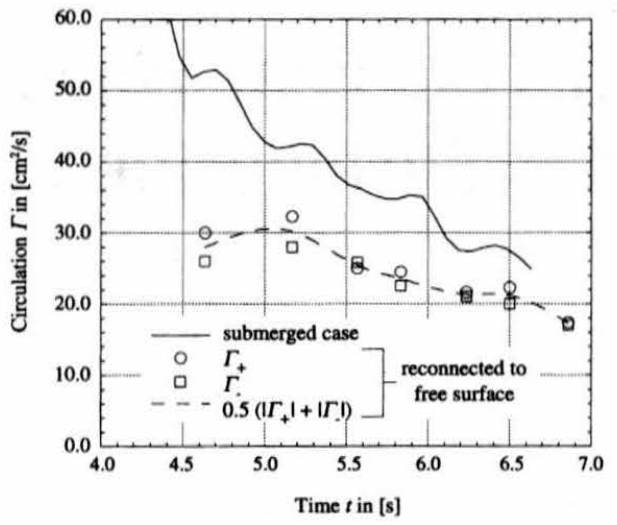

Fig. 12 Comparison of the vortex-ring circulation in the submerged case with the evolution of the total circulation that reconnects to the free surface in the half-plane $z \leq 0$ during the fully-developed turbulent interaction case presented in Fig. 11

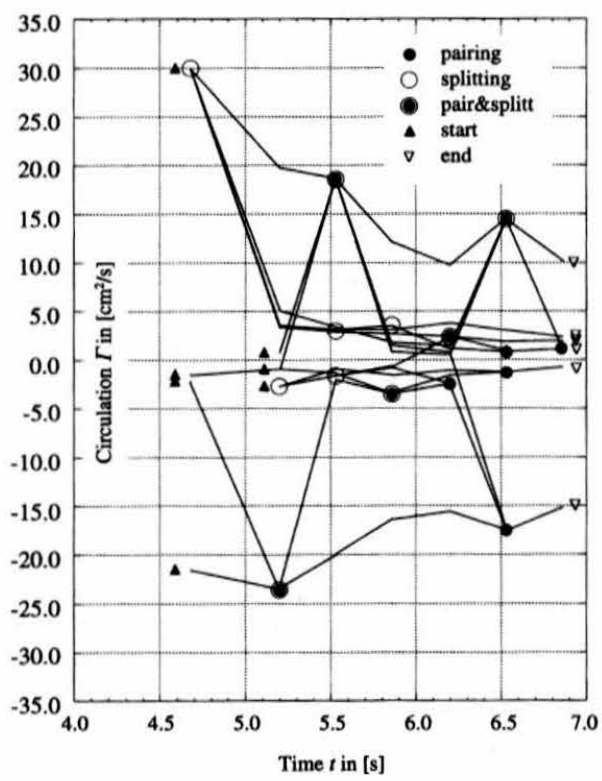

Fig. 13 Temporal evolution of the circulation of individual structures that are reconnected to the free surface in the half-plane $z \leq 0$ during the fully-developed turbulent interaction case presented in Fig. 11

turbulent stage leads also to the bifurcation of the vortex ring. However, from the beginning of the interaction, the bifurcation pattern is superposed by multiple reconnections of small-scale structures. It is interesting to note that the formation of a regular flow pattern at the free surface during the transition and the fully-developed turbulent stage is not affected by the mutual interaction of small-scale structures that are present in the bulk and reconnected to the surface. In combination with relatively high Reynolds numbers, the bifurcation or trifurcation patterns are observed for considerably smaller angles of incidence as compared to the case of laminar vortex-ring/free-surface interactions.
Shadowgraph visualization and simultaneous DPIV measurements of the fully turbulent interaction show that the smallscale structures mutually interact with each other in form of pairing processes that evolve at the surface within the bifurcated vortex-pair pattern. The vorticity field at the free surface is characterized by many pairing and splitting events that evolve on all measured scales. In the case of concentrated and spatially separated vortical structures, the vorticity field is in excellent spatial agreement with the corresponding shadowgraph images.

In addition to the mutual interaction of the small-scale structures, the bifurcated vortex loops strongly align and partially engulf the small-scale structures according to the induced velocity field at the free surface. The latter is dominated by the vortex-pair pattern that contains a relatively high concentration of vorticity and results from the persisting laminar sublayer in the core structure of the turbulent vortex ring.

The dominance of large-scale and highly-concentrated vorticity regions over the small-scale structures at the free surface is similarly observed for the case of turbulent vortex/free surface interactions (see, e.g., Sarpkaya et al., 1994). This behavior can also be expected in higher Reynolds and Froude number flows such as ship wakes, where large-scale structures coexist with small-scale turbulence in the background. Therefore, in terms of modeling turbulent free-surface flows, it is imperative to include the effect of large-scale vortices.

\section{Acknowledgments}

This work has been sponsored by the Office of Naval Research under the ONR-URI grant NO0014-92-J-1610.

\section{References}

Bernal, L. P., and Kwon, J. T., 1989, “'Vortex Ring Dynamics at a Free Surface," Physics of Fluids A, Vol. 1, pp. 449-451.

Bernal, L. P., Hirsa, A., Kwon, J. T., and Willmarth, W. W., 1989, "'On the Interaction of Vortex Rings and Pairs with a Free Surface for Varying Amounts of Surface Active Agents," Physics of Fluids A, Vol. 1, pp. 2001-2004.

Dommermuth, D. G., 1994, "The initialization of vortical free-surface flows," ASME Journal of FLuids EngineERING, Vol. 116, pp. 95-102.

Gharib, M., Weigand, A., Willert, C., and Liepmann, D., 1992, "Experimental Studies of Vortex Reconnection to a Free Surface: A Physical Flow Model,' Preprints, 19th. Symposium on Naval Hydrodynamics, Seoul, Korea, Vol. 2, pp 189-201.

Glezer, A., and Coles, D., 1990, “An Experimental Study of a Turbulent Vortex Ring," Journal of Fluid Mech., Vol. 211, pp. 243.

Krutzsch, C. H., 1936, "Uber ein instabiles Gebiet bei Wirbelringer," Zeitschrift für angewandte Mathematik und Mechanik, Vol. 16, pp. 352-353.

Krutzsch, C. H., 1939, "Über eine experimentell beobachtete Erscheinung an Wirbelringen bei ihrer translatorischen Bewegung in wirklichen Flüssigkeiten,' Annalen der Physik, Vol. 35, pp. 497-523.

Kwon, J. T., 1989, "Experimental Study of Vortex Ring Interaction with a Free Surface," Technical Report No. 89-06, University of Michigan, Dept. of Aerospace Engineering, Ann Arbor, MI.

Maxworthy, T., 1974, "Turbulent Vortex Rings," Journal of Fluid Mechanics, Vol. 64, pp. 227-239.

Sarpkaya, 1992, "Three-Dimensional Interactions of Vortices with a Free Surface," AIAA 92-0059, Proceedings of the 30th AIAA Aerospace Sciences Meeting, Reno, NV

Sarpkaya, T., Magee, M., and Merrill, C., 1994, "Vortices, Free Surface and Turbulence," 1994 ASME Symposium on Free-Surface Turbulence, Lake Tahoe, CA.

Weigand, A., and Gharib, M., 1994, "On the Decay of a Turbulent Vortex Ring," Physics of Fluids, Vol. 6, pp. 3806-3808.

Widnall, S. E., and Sullivan, J. P., 1973, “On the Stability of Vortex Rings," Proceedings of the Royal Society of London, A, Vol. 332, pp. 335-353.

Widnall, S. E., Bliss, D. B., and Tsai, C.-Y., 1974, "The Instability of Short Waves on a Vortex Ring," Journal of Fluid Mechanics, Vol. 66, pp. 35-47.

Willert, C. E., and Gharib, M., 1991, "Digital Particle Image Velocimetry," Experiments in Fluids, Vol. 10, pp. 181-193. 